\title{
Improving care for elderly patients with hip fracture: interdisciplinary collaboration in regional analgesia
}

\author{
Carla Y. Henderson, BM, BS (1) - Ramzy Abdel-Galil, MD • Michael Y. Woo, MD • \\ Jacques S. Lee, MD • Steven Papp, MD • Susan Madden, APRN • \\ Anne Lui, MD
}

Received: 13 March 2019/Revised: 23 March 2019/Accepted: 27 March 2019/Published online: 8 April 2019

(c) Canadian Anesthesiologists' Society 2019

\section{To the Editor,}

Regional analgesia for hip fracture provides superior analgesia, reduces the risk of pneumonia, and decreases time to first mobilization. ${ }^{1}$ Health Quality Ontario strongly recommends the use of regional analgesia for hip fracture. ${ }^{2}$ Unfortunately, ongoing use is generally poor in most institutions, including international jurisdictions., ${ }^{3,4} \mathrm{We}$ implemented an anesthesia-led care pathway for preoperative regional analgesia for hip fracture in June 2015. A practice audit performed from June 2016 to October 2016 found that 18 of 107 (17\%) eligible patients were offered regional analgesia. The goal of this quality improvement (QI) initiative was to identify and mitigate barriers to regional analgesia and increase its availability for hip fracture patients.

Data collection for this Plan-Do-Study-Act (PDSA) evaluation was deemed a QI initiative by the Ottawa Health

C. Y. Henderson, BM, BS $(\bowtie) \cdot$ R. Abdel-Galil, MD

Department of Anesthesiology and Pain Medicine, University of Ottawa, Ottawa, Canada

e-mail: chenderson@toh.ca

M. Y. Woo, MD

Department of Emergency Medicine, The Ottawa Hospital, Ottawa, Canada

\section{J. S. Lee, MD}

Department of Emergency Medicine, Sunnybrook Health

Sciences Center, Toronto, Canada

S. Papp, MD

Department of Orthopedic Surgery, The Ottawa Hospital,

Ottawa, Canada

S. Madden, APRNA. Lui, MD

Department of Anesthesiology and Pain Medicine, The Ottawa

Hospital, Ottawa, ON, Canada
Sciences Network Research Ethics Board (February 2017). Barrier identification and mitigation strategies were solicited from all stakeholder departments through email and meetings. As our institution has resident-led services, input was also gathered in short actionable sessions involving "raise your hand" style surveys and open floor input opportunities from trainees in the departments of orthopedic surgery, anesthesia, and emergency medicine.

Feedback showed a keen interest from all departments in adopting an efficient, integrated pathway to bring regional analgesia to more patients. Areas felt in most need of intervention were increasing the knowledge base in service providers, regional anesthesia skills acquisition, and logistics. Similar barriers have been described elsewhere. ${ }^{4}$ We adopted a multidisciplinary hip fracture regional analgesia service incorporating a hip fracture patient's entire circle of care providers with a newly simplified pathway (Figure).

Emergency medicine physicians were trained to provide femoral nerve blocks as part of the EDU-RAPID multicentre randomized trial (NCT02892968). We also developed a low-fidelity simulation tool for anatomical teaching of a fascia iliaca block. This enabled large-scale peer-to-peer teaching to enhance knowledge base and skills acquisition for residents from all specialties.

Following launch of the new pathway in March 2017, a three-month "wash-in" was allowed before another chart review. From June to September 2017, 38 of 71 (54\%) hip fracture patients were offered regional analgesia. A second PDSA cycle identified that patients transferred to our hospital "direct to service" were not receiving regional analgesia. After consultation with key stakeholders, the absence of assessment by the emergency physician was identified as a barrier. This assessment had typically triggered our pathway. We subsequently recommended 


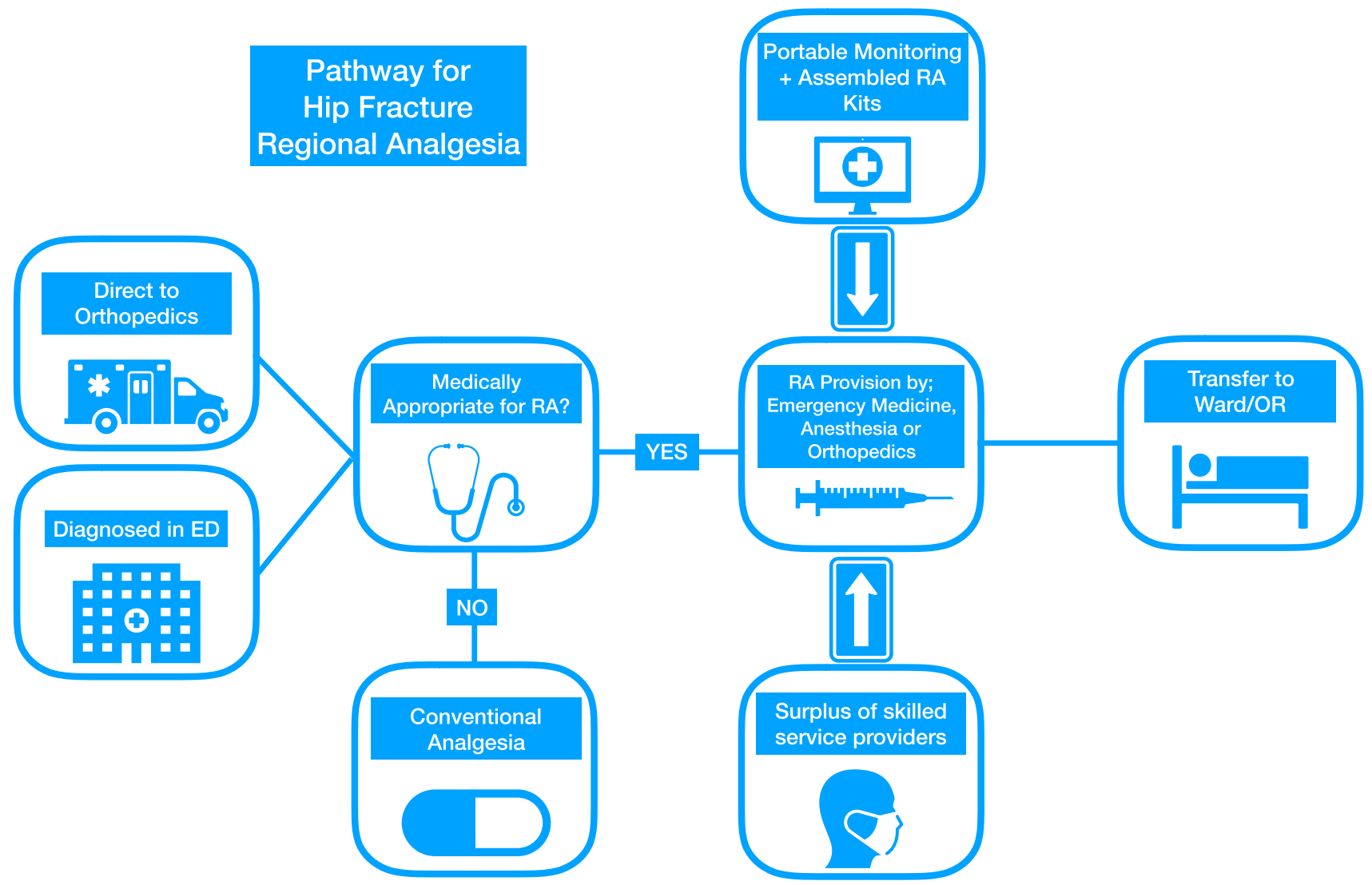

Figure New pathway for hip fracture analgesia management. $\mathrm{ED}=$ emergency department; $\mathrm{OR}=$ operating room; RA = regional analgesia

that the initial assessor, regardless of service, should either provide regional analgesia or enlist the assistance of a colleague from the multidisciplinary team. A third audit from March to June 2018 showed that 65 of 87 (75\%) of hip fracture patients were offered regional analgesia.

Our final pathway simplified the entire process for both providers and patients (see Figure). Following hip fracture diagnosis, regional analgesia is started in the emergency department. Providers are residents/staff from emergency medicine, anesthesia, or orthopedics. Equipment, drugs, and monitoring is readily available in the emergency department.

Recognition from three departments and the hospital that regional analgesia was an essential part of the hip fracture patient's care plan was a critical factor for success. Integration of multidisciplinary skills created a large cohort of skilled physicians, which minimized barriers to care. This QI initiative highlights the need for interdisciplinary collaboration and education in providing quality patient care and provides a model (Figure) that may be applicable to other centres.

\section{Conflicts of interest None declared.}

Editorial responsibility This submission was handled by Dr. Philip M. Jones, Associate Editor, Canadian Journal of Anesthesia.
Funding disclosure No monetary funds were received for this quality improvement initiative.

Data collection was aided by the concurrently running Canadian Institutes of Health Research (CIHR) funded ED Ultrasonographic Regional Anesthesia to Prevent Incident Delirium in Hip Fracture Patients (EDU-RAPID) Multi-center Stepped Wedge Cluster Randomized Trial. No monetary funds were received for this quality improvement initiative.

\section{References}

1. Guay J, Parker MJ, Griffiths R, Kopp S. Peripheral nerve blocks for hip fractures. Cochrane Database Syst Rev 2017; 5: CD001159.

2. Health Quality Ontario; Ministry of Health and Long-Term Care. Quality-Based Procedures: Clinical Handbook for Hip Fracture. Toronto, ON: Health Quality Ontario; May 2013. Available from URL: https://www.hqontario.ca/Portals/0/Documents/evidence/clini cal-handbooks/hip-fracture-130717-en.pdf(accessed March 2019).

3. Youde J, Husk J, Lowe D, Grant R, Potter J, Martin F. The national clinical audit of falls and bone health: the clinical management of hip fracture patients. Injury 2009; 40: 1226-30.

4. Haslam L, Lansdown A, Lee J, van der Vyver M. Survey of current practices: peripheral nerve block utilization by ED physicians for treatment of pain in the hip fracture patient population. Can Geriatr J 2013; 16: 16-21.

Publisher's Note Springer Nature remains neutral with regard to jurisdictional claims in published maps and institutional affiliations. 